\title{
CHANGES OF PLASMA CORTICOTROPHIN RELEASING FACTOR LEVELS IN THE YOUNG RATS WITH HYPOXIC-ISCHEMIC BRAIN DAMAGE
}

\author{
J. Dong ${ }^{1}$, Y.M. Zhu ${ }^{1}$, W.W. Zhou ${ }^{2}$, F. Yuan ${ }^{1}$, C.Y. Dong ${ }^{1}$, Y.H. Gui ${ }^{3}$, C. Chen ${ }^{3}$ \\ ${ }^{1}$ Pediatric Intensive Care Unit, Children's Hospital of Hunan Province, ${ }^{2}$ Department of Chest Surgery, \\ Children's Hospital of Hunan Province, Changsha, ${ }^{3}$ Children's Hospital of Fudan University, Shanghai, \\ China
}

Background and aims: It has been proved that the secreting levels of corticotrophin releasing factor (CRF) were correlated with the hypoxia in some animal experiments, our present study is to explore the changes of plasma CRF levels in the young rats with hypoxic-ischemic brain damage.

Methods: Two hundred forty young rats were randomly divided into twenty-four groups, which each group is ten rats, eight groups were used to build the animal model of hypoxic-ischemic brain damage (model group) to observe the changes of plasma CRF levels in the young rats after different hours (in one day) and different days of hypoxia-ischemia, and eight groups were used as sham operated groups and eight groups were used as normal control groups among these groups. Plasma CRF levels in young rats were measured by radioimmunoassay.

Results: Plasma CRF levels of hypoxia group (model groups) and sham-operated groups and normal control group are no significant difference in the young rats after different hours (in one day) of hypoxia-ischemia; but plasma CRF levels in model groups were significantly lower than those of sham operated groups and normal control groups in young rats after day 1 and day 3 of hypoxia-ischemia $(\mathrm{P}<0.001$ and $\mathrm{P}<0.001$ respectively).

Conclusions: Hypoxia-ischemia affects plasma CRF levels in the young rats, which is related with the time after hypoxia-ischemia. 